\title{
Validação do método analítico em CLAE com derivatização pós-coluna para a quantificação de carbamatos em água de abastecimento público
}

\author{
Validation of the analytical method in HPLC with post-column \\ derivatization for the quantification of carbamates \\ in public water supply \\ Data de entrada: \\ $23 / 08 / 2017$ \\ Data de aprovação: \\ 02/10/2017
}

Alcione Aparecida de Almeida Alves"/ Giselle Louise de Oliveira Ruiz/ Maurício Luiz Sens/Thyara Campos Martins Nonato DOI: 10.4322/dae.2018.024

\section{Resumo}

O objetivo deste estudo foi validar o método analítico em cromatografia líquida de alta eficiência com detector de fluorescência e derivatização pós-coluna para quantificar os carbamatos carbaril, metomil e carbofurano em água de abastecimento público. Os resultados de validação do método garantiram o atendimento às exigências das aplicações analíticas quanto a seletividade, linearidade, intervalo, precisão, exatidão, limite de detecção e limite de quantificação. A aplicação de duas fases móveis e o decréscimo no tempo de detecção dos picos cromatográficos foram considerados aspectos relevantes e positivos, pois auxiliaram na redução de reagentes e no tempo de execução da análise, respectivamente. Palavras-chave: Validação de método. Agrotóxicos. Água de abastecimento.

\section{Abstract}

The objective of this study was to validate the analytical method in high performance liquid chromatography with fluorescence detector and post-column derivatization to quantify the carbamates carbaryl, methomyl and carbofuran in public watersupply. The validation results of the method ensured the requirements of the analytical applications regarding the selectivity, linearity, interval, precision, accuracy, detection limit and quantification limit. The application of two mobile phases and the decrease in the detection time of the chromatographic peaks were considered relevant and positive aspects, as they aided in the reduction of reagents and in the execution time of the analysis, respectively. Keywords: Validation of analytical method. Pesticides. Water supply.

\footnotetext{
Alcione Aparecida de Almeida Alves - Professora da Universidade Federal da Fronteira Sul (UFFS).Doutoranda no Programa de Pós-graduação em Engenharia Ambiental, Universidade Federal de Santa Catarina (UFSC).

Giselle Louise de Oliveira Ruiz - Técnica em Saneamento pelo Instituto Federal de Santa Catarina (IFSC).Graduanda em Engenharia Civil da Universidade Federal de Santa Catarina (UFSC).

Maurício Luiz Sens - Professor Titular no Departamento de Engenharia Sanitária e Ambiental da UFSC. Doutor em Tratamento de Água pela Ecole Nationale Superiéure de Chimie de Rennes (ENSCR).

Thyara Campos Martins Nonato - Doutora em Engenharia Sanitária e Ambiental (UFSC).Pós-doutoranda em Engenharia Ambiental (UFSC).

*Endereço para correspondência: Laboratório de Potabilização de Águas, ENS, CTC, UFSC -Campus Reitor João David Ferreira Lima, s/n -Bairro Trindade, CEP: 88040-970, Florianópolis/Santa Catarina. E-mail: alcione.almeidaauffs.edu.br.
} 


\section{INTRODUÇÃO}

Os carbamatos representam um grupo químico importante de compostos orgânicos que possuem ampla utilidade agrícola e são encontrados nas diferentes subclasses de agrotóxicos, tais como herbicidas, inseticidas e fungicidas (CHATURVEDI, 2012). Dentre os carbamatos, o carbaril, o metomil e o carbofurano pertencem à classe de compostos instáveis derivados do ácido carbâmico (H2N-COOH) (ANVISA, 2017), são classificados como termicamente lábeis e polares e identificados pela presença das ligações O-CO-NH (ADAMS; BARON, 1965).

Esses compostos possuem como principais rotas de contato com ser humano o solo após a sua aplicação nas culturas de algodão, trigo, milho, soja, arroz, feijão, tomate, sorgo, dentre outros (ANVISA, 2017; SALIH et al., 2017), os mananciais hídricos por processo de lixiviação e escoamento superficial (SAXENA et. al., 2014; KENNEDY et al., 2015) e as águas de abastecimento público devido à captação de águas superficiais contaminadas por estes agrotóxicos (DORES; FREIRE, 2001; MARQUUES et al., 2006).

Quando a população é exposta a carbamatos por contato dérmico, inalação ou ingestão, os principais efeitos colaterais estão vinculados ao aumento das secreções corporais, broncoconstrição, hipotensão, contração muscular espasmódica, paralisia muscular e convulsão seguida de depressão do sistema nervoso central (RANG et al., 2004).

Desta forma, quantificar esses compostos, especialmente nas águas de abastecimento público, é primordial, pois o contato direto com solos, plantas ou mananciais hídricos superficiais contaminados pode ser uma opção, diferentemente da ingestão da água, que é a fonte vital de sobrevivência da população humana.

Dentre as técnicas comumente utilizadas nas análises de determinação e quantificação de car- bamatos, a Cromatografia Líquida de Alta Eficiência (CLAE) tem apresentado resultados promissores (OUERTANI et al., 2016; TALEBIANPOOR et al., 2017). Além disso, destaca-se pela alta sensibilidade e seletividade quando acoplada a detectores, como, por exemplo, o detector de fluorescência (KOK et al., 1992; JARDIM et al., 2014).

Para quantificar os carbamatos por CLAE com detector de fluorescência, comumente se realiza o pré-condicionamento da amostra, por meio da extração da fase sólida, a qual visa remover seletivamente resíduos de agrotóxicos em amostras de água e pré-concentrá-los (TANKIEWICZ et al., 2011). Entretanto, esse procedimento pode resultar na degradação térmica de grande parte dos agrotóxicos mais polares, dentre eles, os carbamatos (BERNAL et al. 1992); além disso, essa técnica pode ser considerada uma das etapas mais demoradas e complexas da análise.

Portanto, para evitar a degradação desses compostos, reduzir o tempo de realização da análise e quantificar os carbamatos em baixas concentrações (de $\eta \mathrm{g} \mathrm{L}^{-1}$ a $\mu \mathrm{g} \mathrm{L}^{-1}$ ), a derivatização pós-coluna tem sido empregada junto à CLAE com detector de fluorescência (USEPA, 2001).

Nesse método analítico, o derivatizador pós-coluna precedido por um cromatógrafo a líquido hidrolisa os analitos (carbamatos) e, por meio de uma reação com $\mathrm{NaOH}$, forma a metilamina, que ao reagir o com o-ftaldeído (OPA) e o 2-mercaptoetanol forma um composto fluorescente, o l-hidroxiletiltiol-2-metilisoindole, que pode ser identificado no detector de fluorescência do cromatógrafo ( $\mathrm{CHI}-$ RON; BARCELÓ, 1993; USEPA, 2001).

Durante a realização desta análise, usualmente ocorre a aplicação de três fases móveis no cromatógrafo a líquido, as quais são responsáveis por detectar seletivamente os carbamatos metomil, carbofurano e carbaril em aproximadamente 7 , 15 e 18 min. de reação e evitar a sobreposição dos picos cromatográficos (USEPA, 2001). 
Para Neto (2010), a existência de picos sobrepostos num cromatograma indica nitidamente a coeluição de um interferente que compartilha a mesma massa/carga ou a mesma transição do composto de interesse. $\mathrm{Ou}$, em se tratando de compostos distintos, a sobreposição dos picos cromatográficos poderá ser ocasionada pela relação número de analitos $\uparrow$ versus tempo de análise $\downarrow$.

Desta forma, se o número de carbamatos a serem quantificados for reduzido, a sobreposição dos picos cromatográficos poderá ser evitada, apesar da redução do tempo de análise e de uma fase móvel junto ao cromatógrafo.

Sendo assim, os grandes desafios para o aprimoramento desse método analítico consistem em reduzir a utilização de reagente aplicado à fase móvel e otimizar o tempo de análise, evitando a sobreposição de picos cromatográficos. E, para garantir a qualidade e a confiabilidade dos resultados obtidos, bem como superar esses desafios, torna-se imprescindível a validação do método cromatográfico.

No Brasil, os órgãos que regulamentam a validação de métodos analíticos são a Agência Nacional de Vigilância Sanitária (Anvisa, 2003) e o Instituto Nacional de Metrologia, Normalização e Qualidade Instrumental (Inmetro, 2010).

De acordo com a Resolução $N^{\circ} 899 / 2003$ da Anvisa, a validação do procedimento de medição (VPM) deverá garantir por meio de estudos experimentais que o método atenda às exigências das aplicações analíticas, assegurando dessa forma a confiabilidade dos resultados obtidos.

Os parâmetros de validação de métodos analíticos deverão contemplar a seletividade, linearidade, intervalo, precisão, exatidão, limite de detecção
(LD) e limite de quantificação (LQ) (ANVISA, 2003). Esses parâmetros deverão ser aplicados durante o desenvolvimento de um método ou após a realização de modificações de metodologias reconhecidas e/ou aceitas internacionalmente.

Nesse sentido, o objetivo principal deste estudo foi validar o método analítico em CLAE com derivatização pós-coluna para quantificar os carbamatos carbaril, metomil e carbofurano em água de abastecimento.

\section{METODOLOGIA}

\subsection{Reagentes e solventes}

Os reagentes e solventes de grau analítico utilizados neste estudo foram os carbamatos carbaril, metomil e carbofurano (Sigma-Aldrich $®)$; a água ultrapura (MilliQ@) e a acetonitrila (J.T.Backer $\left.{ }^{\circledR}\right)$ empregadas na fase móvel; o metanol (Merck $®)$, o 2-mercaptoetanol (Merck $®)$, o ácido bórico (Sigma-Aldrich $®$ ), o OPA (Sigma-Aldrich $\circledast$ ) e o hidróxido de sódio (Synth $®)$ aplicados junto ao derivatizador na reação pós-coluna para formação do composto fluorescente a ser detectado no detector de fluorescência do cromatógrafo a líquido (Thermo Scientific - Dionex UltiMate 3000).

As soluções contendo carbaril, metomil e carbofurano em concentrações de 0,5 e $100 \mathrm{\mu g} \mathrm{L}^{-1}$ foram preparadas imediatamente antes do uso, por meio de diluição da solução padrão desses carbamatos em acetonitrila.

$\mathrm{Na}$ Tabela 1 estão descritas informações acerca das propriedades físico-químicas e toxicológicas, bem como os principais produtos de degradação dos agrotóxicos analisados neste estudo. 
Tabela 1 - Propriedades físico-químicas dos carbamatos carbaril, metomil e carbofurano.

\begin{tabular}{|c|c|c|c|c|}
\hline Carbamatos & Estrutura química & $\begin{array}{l}\text { Massa Molar } \\
\left(\mathrm{g} \mathrm{mol}^{-1}\right)\end{array}$ & $\begin{array}{l}\mathrm{DL}_{\text {50ratos }} \\
\left(\mathrm{mg} \mathrm{kg}^{-1}\right)\end{array}$ & $\begin{array}{l}\text { Principal produto de } \\
\text { degradação }\end{array}$ \\
\hline Carbaril & 1-naphthyl methylcarbamate & 201,2 & 1 & 1-naftol \\
\hline Metomil & $\begin{array}{c}\mathrm{CH}_{3} \mathrm{NHCO}_{2} \mathrm{~N}=\mathrm{C}-\mathrm{SCH}_{3} \\
\substack{\text { S-methyl N- (methylcarbamoyloxy) } \\
\text { thioacetimidate }}\end{array}$ & 162,2 & 21 & $\begin{array}{l}\text { Metomil metiol } \\
\text { Sulfoxide oxime }\end{array}$ \\
\hline Carbofurano & 2,3-dihydro-2,2-dimethylbenzofuran-7-yl & 221,3 & 8 & $\begin{array}{l}\text { 3-Cetocarbofuran } \\
\text { 3-Hidroxicarbofuran }\end{array}$ \\
\hline
\end{tabular}

Nota: (DL) Dose letal. Fonte: Agência Nacional de Vigilância Sanitária (2003).

\subsection{Matriz Aquosa}

A água de abastecimento público, denominada "água tratada por sistema convencional" (ATSC) utilizada na validação do método analítico proposto por este estudo, foi proveniente da mistura de águas dos rios Cubatão Sul e Vargem do Braço, localizados no Estado de Santa Catarina. Na sequência, a água foi potabilizada por meio do tratamento convencional realizado na Estação de Tratamento de Água (ETA) José Pedro Horstmann (Morro dos Quadros), administrada pela Companhia Catarinense de Águas e Saneamento (CASAN) e localizada no município de Palhoça-SC.

O tratamento convencional contemplou as etapas de coagulação com Policloreto de Alumínio (PAC), filtração rápida por meio de filtros ascendentes (areia), correção de pH com óxido de cálcio $(\mathrm{CaO})$, desinfecção por cloro gasoso $\left(\mathrm{Cl}_{2}\right)$, fluoretação por meio da adição de fluorsilicato de sódio (NaSiF6) e reservação (CASAN, 2013).

Após a reservação, a ATSC foi coletada no Laboratório de Potabililização de Água (LAPOÁ) na
Universidade Federal de Santa Catarina (UFSC) e fortificada com os carbamatos carbaril, metomil e carbofurano.

\subsection{Instrumentação e procedimentos}

Os procedimentos realizados para a quantificação de carbamatos foram descritos no Método 531.2 (USEPA, 2001). Entretanto, fez-se a remoção da fase móvel composta por metanol.

Todas as amostras de ATSC, bem como os reagentes aplicados à fase móvel foram previamente filtradas em membranas em Fluoreto Polivinidileno (PVDF) de 0,22 $\mu \mathrm{m}$ de poro.

Após a filtração, fez-se a injeção de $1000 \mu \mathrm{L}$ de carbaril, metomil e carbofurano, por meio de um amostrador automático no cromatógrafo a líquido (Thermo Scientific - Dionex UltiMate 3000), equipado com coluna de fase reversa (cartuchos de octadecil - C18). As reações ocorreram sob $30^{\circ} \mathrm{C}$ de temperatura e fluxo de $1,0 \mathrm{~mL} \mathrm{~min}^{-1}$. 
Após a passagem pela coluna de fase reversa $\mathrm{C} 18$, os analitos foram hidrolisados em uma reação de derivatização pós-coluna (Pickering PCX $5200 \AA$ ), com $0,075 \mathrm{~N}$ de $\mathrm{NaOH}$ a $80{ }^{\circ} \mathrm{C}$ para formar-se a metilamina, que ao reagir com o OPA e mercaptaetanol produziu o I-hidroxiletiltiol-2-metilisoindole, sendo este composto fluorescente e, portanto, identificável no detector de fluorescência do cromatógrafo (emissão a 340 nm/465 nm).

$\mathrm{Na}$ fase móvel, empregada para separação dos picos de absorção, foram utilizados água ultrapura e acetonitrila. Esses sistemas de gradientes de solventes foram mantidos entre $30 \%$ e $100 \%$, durante aproximadamente 16 minutos de análise.

Para a construção da curva de calibração (CC), utilizou-se o procedimento de padronização externa, o qual tem por característica comparar a área da substância quantificada na amostra com as áreas obtidas em análises de soluções com concentrações conhecidas e preparadas a partir de um padrão.

E, a fim de possibilitar a validação do método analítico em CLAE com derivatização pós-coluna para a quantificação de carbamatos em água de abastecimento público, os procedimentos descritos na Resolução N 899/2003 da ANVISA, respectivos a seletividade, linearidade, intervalo, precisão, LD, LQ e exatidão, foram realizados.

\subsection{Seletividade}

A seletividade se refere à capacidade que um método possui em medir exatamente um composto na presença de outros elementos, tais como impurezas, produtos de degradação e componentes inerentes à matriz (Anvisa, 2003).

Para prever a seletividade do método, realizou-se a análise de quantificação de carbamatos na ATSC e fortificada com $25 \mu \mathrm{g} \mathrm{L}-1$ de carbaril, metomil e carbofurano, seguido da análise da ATSC sem a presença de carbamatos para, dessa forma, identificar a presença ou ausência de sobreposição de picos cromatográficos por quaisquer outros compostos existentes na ATSC, bem como demonstrar não haver sobreposição entre os picos cromatográficos dos carbamatos analisados.

\subsection{Linearidade e Intervalo}

A capacidade de uma metodologia analítica de demonstrar que os resultados obtidos são diretamente proporcionais à concentração do analito na amostra dentro de um intervalo especificado corresponde à linearidade (Anvisa, 2003).

Sendo assim, a linearidade foi averiguada por meio de três CC com seis concentrações, correspondentes a $0,5,1,5,10,25,50$ e $100 \mu \mathrm{g} \mathrm{L}^{-1}$ de carbaril, metomil e carbofurano e atestou-se como linearmente aceita a CC com o coeficiente de correlação $\left(r^{2}\right) \geq 0,99$.

O intervalo, que remete à faixa entre os limites de quantificação superior e inferior do método analítico, também foi definido pela CC.

\subsection{Precisão}

A precisão expressa a proximidade dos resultados obtidos em uma série de medições referente a uma mesma amostra (Anvisa, 2003).

A precisão foi considerada sob duas formas: (i) a precisão intra-corrida por meio da injeção dos padrões de carbaril, metomil e carbofurano em três níveis de concentração, baixo $(0,5 \mu \mathrm{g} L-1)$, médio (50 $\mu \mathrm{g} \mathrm{L-1)}$ e alto (100 $\mu \mathrm{g} \mathrm{L-1)} \mathrm{e;} \mathrm{(ii)} \mathrm{a} \mathrm{precisão} \mathrm{inter-}$ -corridas, correspondente a três injeções consecutivas de cada nível de concentração (baixo, médio e alto), com repetição durante dois dias distintos.

A precisão desse método analítico foi expressa considerando o desvio padrão relativo (DPR), conforme apresentado na Equação 1:

$$
\mathrm{D}_{\mathrm{RP}}=\left(\frac{\mathrm{D}_{\mathrm{P}}}{\mathrm{C}_{\mathrm{MD}}}\right) 100
$$


Em que: $\left(D_{R P}\right)$ desvio padrão relativo (\%); $\left(D_{P}\right)$ desvio padrão $\left(\mu \mathrm{g} \mathrm{L}^{-1}\right) ;\left(\mathrm{C}_{\mathrm{MD}}\right)$ concentração média determinada $\left(\mu \mathrm{g} \mathrm{L}^{-1}\right)$.

Para a validação do método analítico quanto à precisão, foram admitidos valores $<15 \%$, conforme determinação prevista na Resolução $\mathrm{N}^{\circ}$ 899/2003 da Anvisa.

\subsection{Limite de detecção (LD)}

O LD representa a menor quantidade do analito presente em uma amostra que pode ser detectado, entretanto não necessariamente quantificado sob as condições experimentais estabelecidas (Anvisa, 2003). O LD foi calculado considerando os parâmetros da CC e expresso de acordo com a Equação 2.

$$
\mathrm{LD}=\frac{\left(\mathrm{DP}_{\mathrm{a}}\right) 3}{\mathrm{IC}}
$$

Em que: (LD) limite de detecção ( $\left.\mu \mathrm{g} \mathrm{L}^{-1}\right)$; (DPa) desvio padrão do intercepto com o eixo do $Y$ de, no mínimo, 3 curvas de calibração construídas, contendo concentrações do analito próximas ao suposto limite de quantificação ( $\left.\mu \mathrm{g} \mathrm{L}^{-1}\right)$; (IC) inclinação da curva de calibração.

\subsection{Limite de quantificação (LQ)}

O LQ corresponde à menor quantidade do analito em uma amostra que pode ser determinada com precisão e exatidão aceitáveis sob as condições experimentais estabelecidas (ANVISA, 2003). Assim como o LD, o LQ foi calculado considerando os parâmetros da CC, sendo expresso de acordo com a Equação 3.

$$
L Q=\frac{\left(D_{a}\right) 10}{I C}
$$

Em que: (LQ) limite de quantificação $\left(\mu \mathrm{g} \mathrm{L}^{-1}\right)$; (DPa) desvio padrão do intercepto com o eixo do Y de, no mínimo, três curvas de calibração con- tendo concentrações do analito próximas ao suposto limite de quantificação ( $\left.\mu \mathrm{g} \mathrm{L}^{-1}\right)$; (IC) inclinação da curva de calibração.

\subsection{Exatidão}

A exatidão consiste no grau de concordância entre um valor medido e um valor verdadeiro de um mensurando (Inmetro, 2012).

Assim, para a validação do método analítico proposto neste estudo, a exatidão foi expressa pela relação entre a concentração média determinada experimentalmente e a concentração teórica, sendo verificada a partir de nove determinações, com três concentrações (baixa, média e alta) e três réplicas cada.

A exatidão foi considerada dentro dos limites de averiguação da CC, associada aos valores de precisão e descrita por meio da Equação 4.

$\mathrm{E}=\left(\frac{\mathrm{C}_{\mathrm{me}}}{\mathrm{C}_{\mathrm{T}}}\right) 100$

Em que: $(\mathrm{E})$ exatidão; $\left(\mathrm{C}_{\mathrm{me}}\right)$ concentração média experimental $\left(\mu \mathrm{g} \mathrm{L}^{-1}\right),\left(\mathrm{C}_{\mathrm{t}}\right)$ concentração teórica $\left(\mu \mathrm{g} \mathrm{L}^{-1}\right)$.

\section{RESULTADOS E DISCUSSÃO}

Na Figura 1a, referente ao cromatograma da ATSC e fortificada com carbamatos à $25 \mu \mathrm{g} \mathrm{L}^{-1}$, pode-se observar a detecção dos picos cromatográficos em 4,763 min (carbaril), 9,983 min (metomil) e $10,613 \mathrm{~min}$ (carbofurano).

Segundo a USEPA (2001), por meio da CLAE com detecção de fluorescência e derivatização pós-coluna obtêm-se os picos cromatográficos em 7,323 min (metomil), 15,842 min (carbaril) e $18,402 \mathrm{~min}$ (carbofurano).

Logo, o tempo de detecção desses carbamatos foi inferior ao descrito pela USEPA (2001); consequentemente houve a redução do tempo total de realização da análise. 

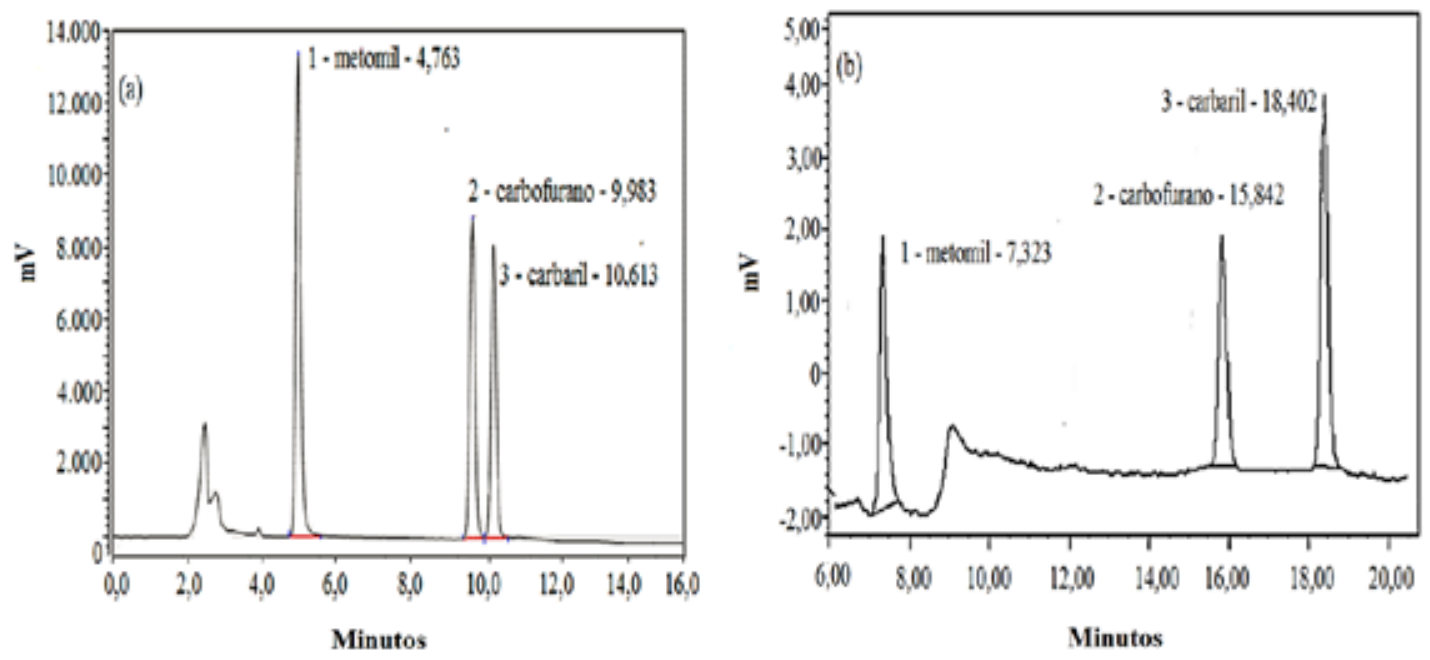

Figura 1 - Detecção dos picos cromatográficos correspondentes ao carbaril, metomil e carbofurano. (a) cromatograma obtido por este estudo; (b) cromatograma obtido pela USEPA (2001).

Constatou-se ainda, por meio dos cromatogramas apresentados nas Figuras $2 \mathrm{a}\left(250 \mathrm{\mu g} \mathrm{L}^{-1}\right) \mathrm{e}$ $2 \mathrm{~b}\left(500 \mu \mathrm{g} \mathrm{L}^{-1}\right)$, que o aumento da concentração dos carbamatos na ATSC, considerando o menor tempo de detecção se comparado à USEPA (2001), não ocasionou a sobreposição dos picos cromatográficos.

Entretanto, o metomil (Figura 1d), apesar de detectado, não foi quantificado, pois a altura do pico cromatográfico foi superior ao valor máximo do eixo y. Sendo assim, a detecção dos picos cromatográficos foi limitada pela concentração desses compostos na ATSC, mas não foi identificada qualquer relação entre a concentração dos carbamatos e a sobreposição dos picos cromatográficos, mesmo após o aumento da concentração de carbaril, metomil e carbofurano em 1.000 e 2.000 $\%$, Figuras 1c e 1d, respetivamente.
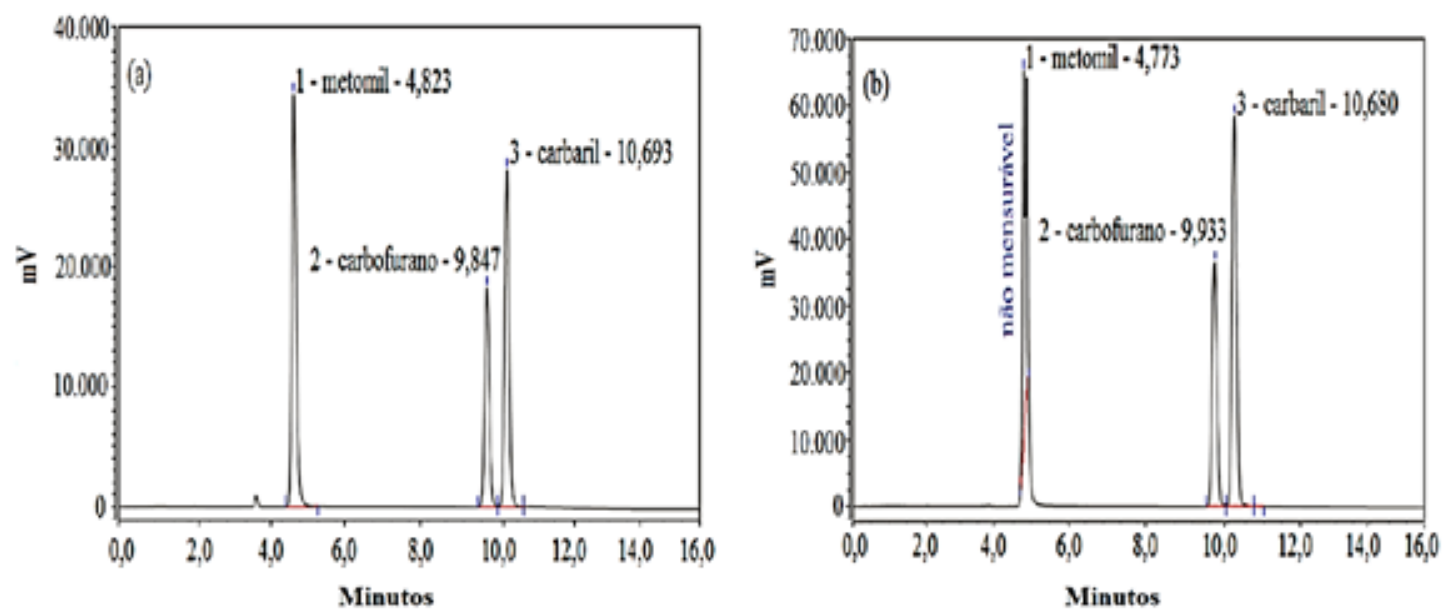

Figura 2 - Detecção dos picos cromatográficos correspondentes ao carbaril, metomil e carbofurano.

(a) concentração de $250 \mu \mathrm{g} \mathrm{L}^{-1}$; (b) concentração de $500 \mu \mathrm{g} \mathrm{L}^{-1}$. 
Essa redução em relação ao tempo de detecção dos picos cromatográficos também pode ser constatada por meio da comparação dos resultados obtidos em alguns dos principais estudos de quantificação de carbaril, metomil e carbofurano, por meio do método analítico em CLAE com derivatização pós-coluna, conforme apresentado na Tabela 2.

Tabela 2 - Algumas das principais pesquisas de determinação de carbamatos por meio da CLAE com detector de fluorescência e derivatização pós-coluna.

\begin{tabular}{|c|c|c|c|}
\hline \multicolumn{3}{|c|}{ Tempo de detecção (min) } & \multirow{2}{*}{ Autores } \\
\hline Metomil & Carbofurano & Carbaril & \\
\hline 10,67 & 21,75 & 22,82 & $\begin{array}{l}\text { KOK et al. (1990) } \\
\text { KOK et al. (1992) }\end{array}$ \\
\hline NA & $\approx 48$ & $\approx 55$ & $\begin{array}{c}\text { Chiron; Barceló } \\
\text { (1993) }\end{array}$ \\
\hline 6,197 & 11,325 & 12,038 & McGarvey (1994) \\
\hline 7,323 & 15,842 & 18,402 & USEPA (2001) \\
\hline$\approx 9$ & $\approx 11$ & $\approx 12$ & Nogueira et al. (2003) \\
\hline NA & $\approx 19$ & $\approx 21$ & KOC et al. (2008) \\
\hline 4,763 & 9,983 & 10,613 & EP \\
\hline
\end{tabular}

Nota: (NA) não analisado; (EP) esta pesquisa.

Considerando a Tabela 2 e as Figuras $1 \mathrm{ab}$ e $2 \mathrm{ab}$, pode-se afirmar que a boa resolução dos picos cromatográficos no menor intervalo de tempo é considerada neste estudo um fator positivo, porque propiciou a redução do tempo total da análise. Consequentemente, obtém-se redução de custos, pois o reagente OPA aplicado junto ao derivatizador pós-coluna é considerado estável durante um período de 36 horas (USEPA, 2001), ou seja, quanto menor o tempo de realização de uma análise, maior o número de amostras analisadas num intervalo de 1,5 dia.

A obtenção de resolução suficiente para que ocorresse a separação de todos os picos cromatográficos no menor tempo de análise, a inexistência de sobreposição destes e o tempo de detecção do primeiro analito próximo ao eixo $y$, mas sem o comprometimento quanto a sua quantificação, está relacionado à não saturação da coluna $\mathrm{C} 18$ de fase reversa, bem como à força cromatográfica e a seletividade das fases móveis aplicadas à CLAE (SILVA; COLLINS, 2011).

Dessa forma, considera-se que a retirada de uma fase móvel (metanol) não influenciou negativamente quanto à detecção dos picos cromatográficos dos carbamatos carbaril, metomil e carbofurano, possibilitando ainda a eliminação de uso deste reagente durante a análise.

Entretanto, deve-se ressaltar que, quanto maior o número de analitos a serem detectados em uma amostra, mais facilmente ocorre a sobreposição dos picos cromatográficos, fazendo-se necessário, por vezes que todas as fases móveis sugeridas por uma metodologia sejam aplicadas.

\subsection{Seletividade}

Os cromatogramas respectivos à ATSC sem carbamatos (Figura 3a), após a fortificação (25 $\mathrm{g} \mathrm{L} \mathrm{L}^{-1}$ ) com carbaril, metomil e carbofurano (Figura 3b), demonstraram a seletividade do método, ou seja, a sua capacidade de medir exatamente os carbamatos na presença de outros possíveis componentes existentes na água de abastecimento público.

Tal constatação demonstrou que as características intrínsecas da ATSC, tais como cor, turbidez e a presença de matéria orgânica natural, não influenciaram a definição dos picos cromatográficos. Assim, a seletividade do método foi comprovada, pois este foi capaz determinar os analitos de maneira inequívoca na presença de outras substâncias susceptíveis de interferirem nessa determinação (LANÇAS, 2004). 

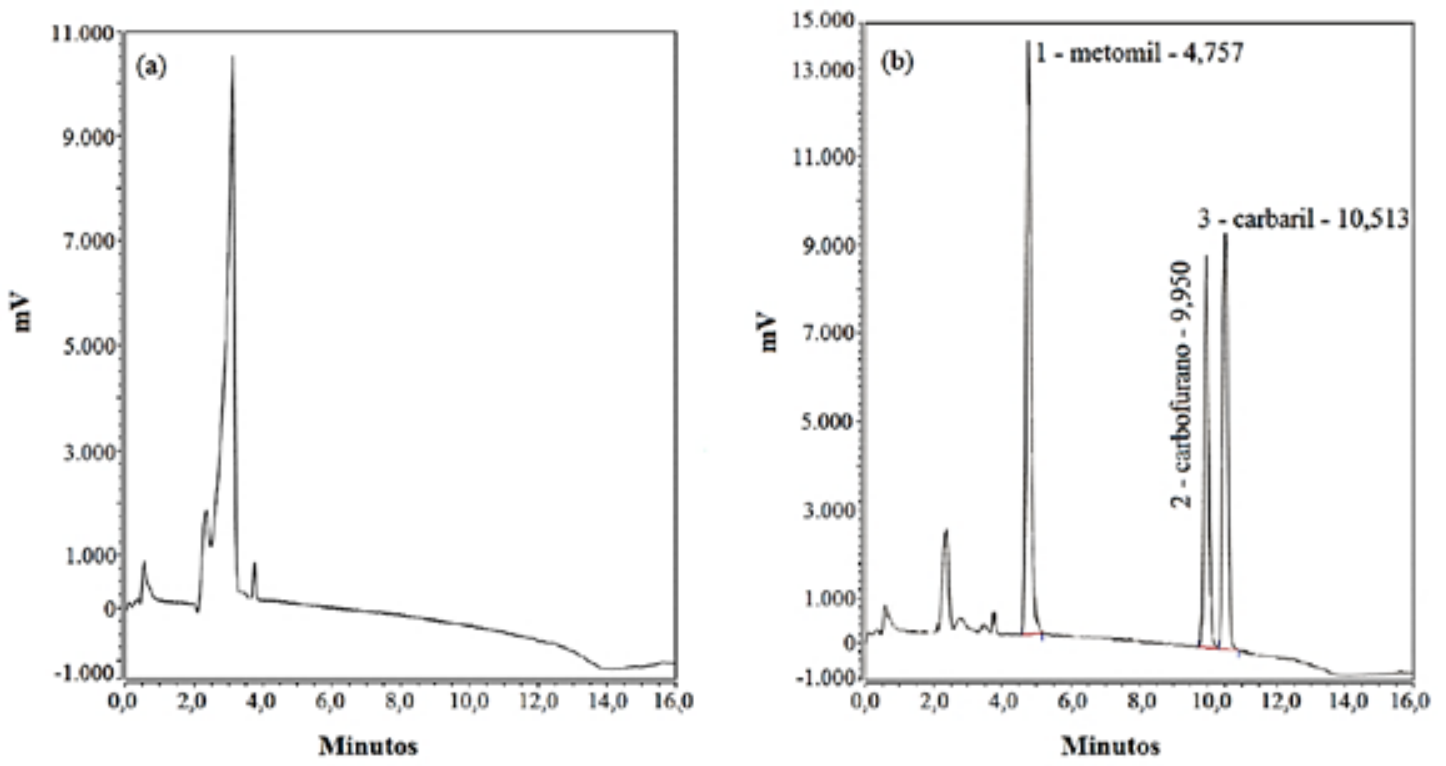

Figura 3 - Cromatograma da água tratada por sistema convencional (a) ATSC sem carbamatos; (b) ATSC fortificada com carbaril, metomil e carbofurano.

Os diferentes tempos de detecção dos picos cromatográficos (Figura 3b) referentes ao metomil, carbofurano e carbaril atestaram não haver sobreposição entre os analitos de interesse, estando, portanto separados entre si e dos demais compostos presentes na amostra.

Nas Figuras 3a e 3b, pode-se constatar ainda, a presença de um composto desconhecido (tempo $<4$ minutos), no entanto tal substância inerente à ATSC não influenciou nem se sobrepôs aos picos cromatográficos referentes aos carbamatos.

\subsection{Linearidade e intervalo}

A linearidade do método foi comprovada por meio dos valores dos coeficientes de correlação linear (CCL) de três curvas de calibração (Figuras 4a, 4b e 4c), referentes aos carbamatos carbaril, metomil e carbofurano, respectivamente. A faixa linear de trabalho, também denominada intervalos da CC, foi de $0,5 \mu \mathrm{g} \mathrm{L}^{-1}$ a $100 \mu \mathrm{g} \mathrm{L}^{-1}$.

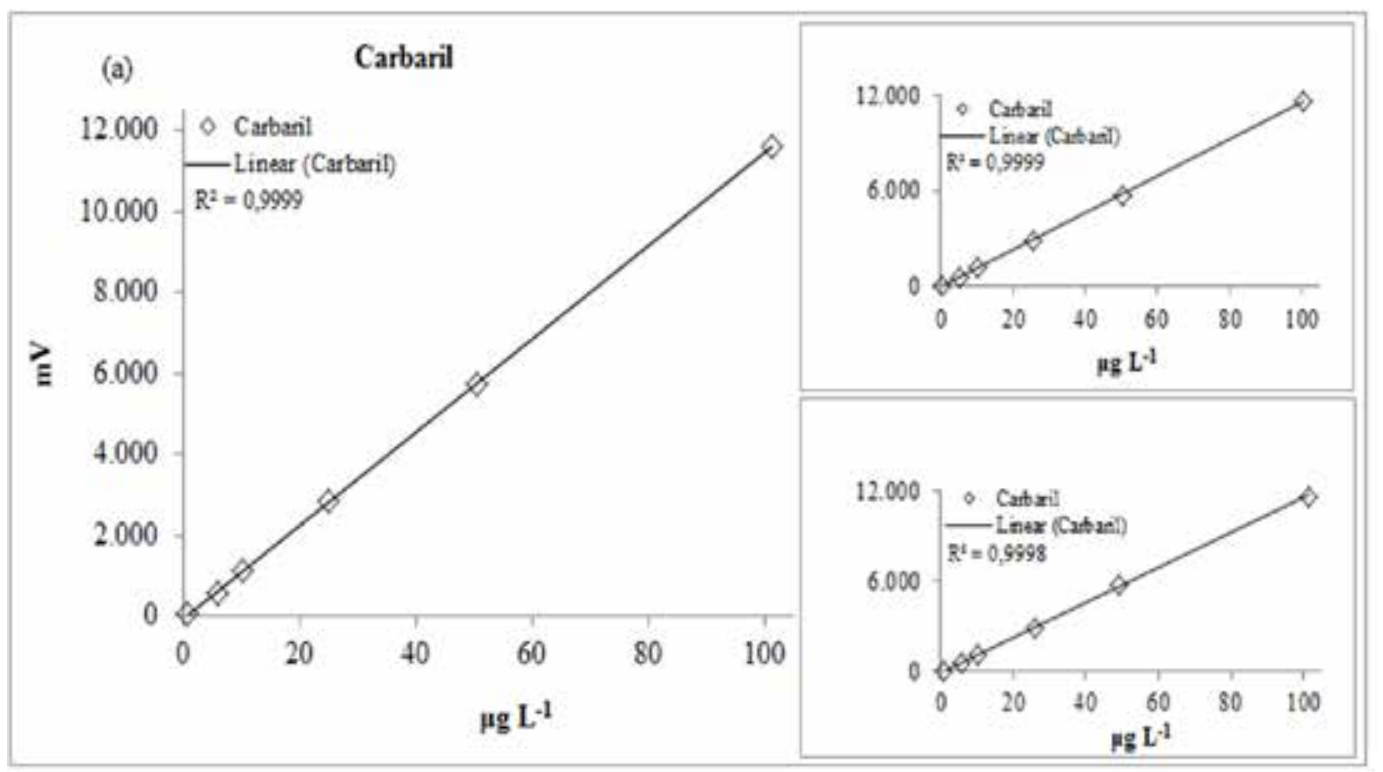



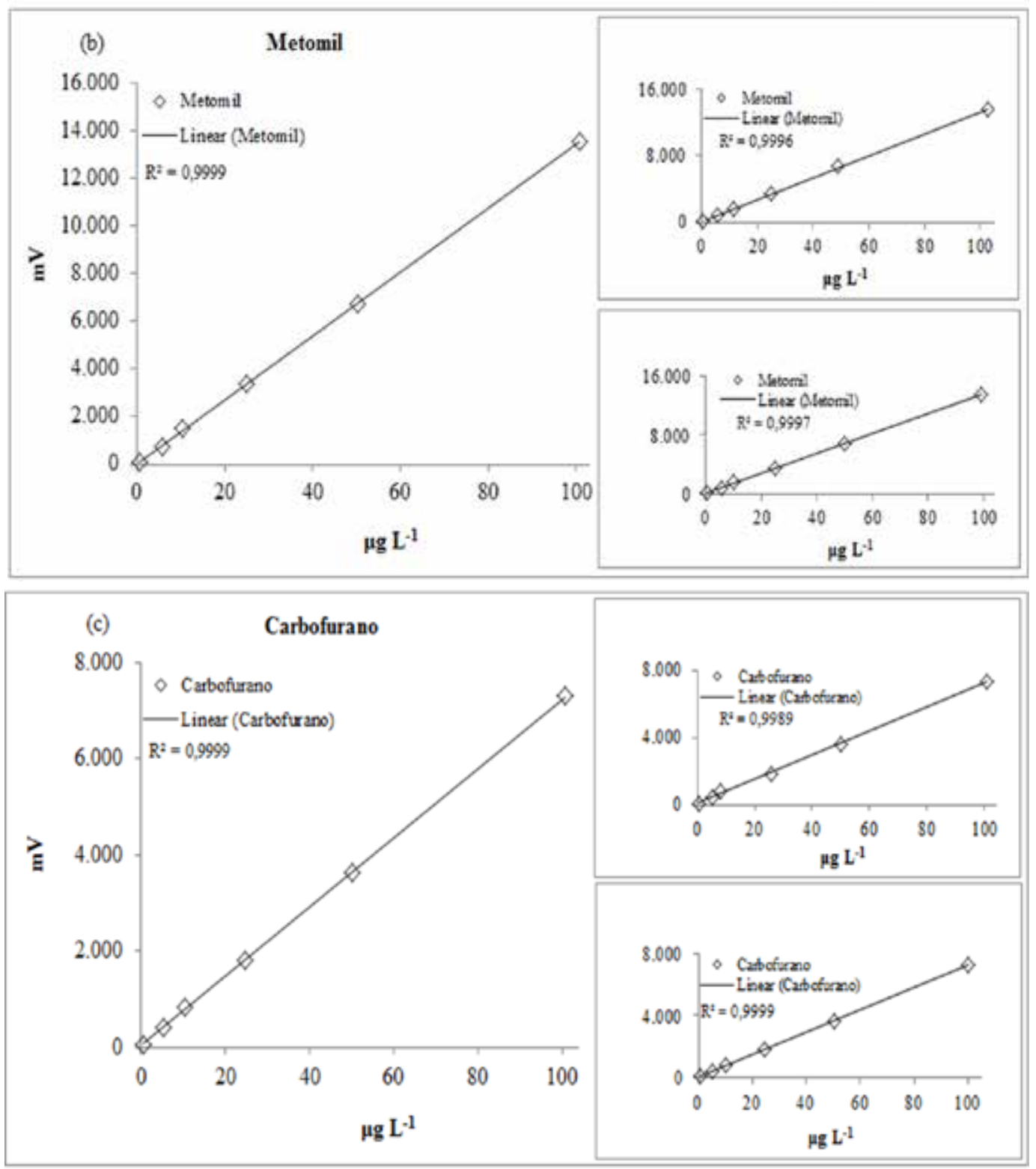

Figura 4 - Curvas de calibração dos carbamatos [0,5 a $\left.100 \mu \mathrm{g} \mathrm{L}^{-1}\right]$, com seus respectivos coeficientes de correlação linear; (a) carbaril; (b) metomil; (c) carbofurano.

A Anvisa (2003) recomenda um CCL igual a 0,99 e o lnmetro (2010), um valor acima de 0,90. De acordo com a Figura 4a-c, o CCL das CC respectivas aos carbamatos atendeu aos valores mínimos estabelecidos pelos órgãos supracitados, sendo todos superiores a 0,99.

Somente a segunda CC, referente ao carbofurano, o CCL foi inferior a 0,999 (0,9989); todos os demais foram superiores, atestando desta forma a linearidade do método.
Segundo Shabir (2003), um CCL maior que 0,999 é entendido como uma evidência de um ajuste ideal dos dados, e quanto mais próximo de 1,0, menor a dispersão do conjunto de pontos experimentais.

Os resultados obtidos em relação à linearidade do método analítico em CLAE com derivatização pós-coluna corroboram o descrito por Kok et al. (1992). Naquele estudo, a linearidade foi considerada satisfatória, pois apresentou $C C L$ entre 0,995 
e 1,0 para a determinação de carbamatos, dentre eles o carbaril e o carbofurano.

Resultados similares de linearidade e intervalo foram descritos por Parreira et al. (2001), os quais realizaram a validação de método analítico para a determinação simultânea de carbaril, carbofurano e metomil, dentre outros agrotóxicos, em amostras de águas natural e potável e obtiveram um CCL igual a 0,9999 para o intervalo de concentrações entre 0,50 e $100,00 \mu \mathrm{g} \mathrm{L}^{-1}$.

\subsection{Precisão e exatidão}

A precisão inter-corridas e intra-corridas, expressas por meio do DPR, e a exatidão do método analítico podem ser averiguados na Tabela 3. Nesta encontram-se descritos os resultados das médias de nove medições, realizadas em um dia e replicadas no dia seguinte, considerando três níveis de concentração: baixa $\left(0,5 \mu \mathrm{g} \mathrm{L}^{-1}\right)$, média $\left(50 \mu \mathrm{g} \mathrm{L}^{-1}\right)$ e alta $\left(100 \mu \mathrm{g} \mathrm{L}^{-1}\right)$ referentes à ATSC e fortificada com os carbamatos carbaril, metomil e carbofurano.

Tabela 3 - Avaliação da precisão e exatidão do método para análise de carbaril, metomil e carbofurano por CLAE com detector de fluorescência e derivatização pós-coluna.

\begin{tabular}{|c|c|c|c|c|c|c|c|}
\hline \multirow{2}{*}{ Analito } & \multirow{2}{*}{ Medições* } & \multicolumn{3}{|c|}{ Primeiro dia } & \multicolumn{3}{|c|}{ Segundo dia } \\
\hline & & Baixa & Média & Alta & Baixa & Média & Alta \\
\hline \multirow{13}{*}{ Carbaril } & 1 & 0,5219 & 48,4982 & 100,4955 & 0,5104 & 50,1057 & 100,4910 \\
\hline & 2 & 0,5019 & 48,4982 & 100,2940 & 0,5116 & 50,1082 & 100,3980 \\
\hline & 3 & 0,5104 & 49,8839 & 100,4955 & 0,5295 & 50,1129 & 100,3988 \\
\hline & 4 & 0,4968 & 49,8982 & 101,2020 & 0,5295 & 52,1038 & 100,3988 \\
\hline & 5 & 0,5103 & 49,8839 & 101,3045 & 0,5295 & 52,1038 & 100,9039 \\
\hline & 6 & 0,4930 & 49,2949 & 101,2020 & 0,5367 & 51,9583 & 101,3918 \\
\hline & 7 & 0,5034 & 49,2850 & 100,4392 & 0,5367 & 51,4820 & 101,4243 \\
\hline & 8 & 0,5029 & 49,2850 & 100,0593 & 0,5268 & 51,4820 & 101,4243 \\
\hline & 9 & 0,5103 & 50,2945 & 99,5920 & 0,4968 & 51,4820 & 100,7857 \\
\hline & $\sigma\left(\mu \mathrm{g}^{-1}\right)$ & 0,5034 & 49,2949 & 100,4955 & 0,5295 & 51,4820 & 100,7857 \\
\hline & $\left(\mu \mathrm{g} \mathrm{L}^{-1}\right)$ & 0,0086 & 0,6300 & 0,5766 & 0,0137 & 0,8672 & 0,4610 \\
\hline & DPR (\%) & 1,7129 & 1,2780 & 0,5738 & 2,5789 & 1,6845 & 0,4574 \\
\hline & Exatidão & 100,68 & 98,58974 & 100,4955 & 105,9 & 102,964 & 100,7857 \\
\hline \multirow{13}{*}{ Metomil } & 1 & 0,5028 & 49,8969 & 102,8408 & 0,5190 & 49,9133 & 99,5939 \\
\hline & 2 & 0,4928 & 49,8969 & 102,8408 & 0,5111 & 50,1980 & 99,5939 \\
\hline & 3 & 0,5158 & 50,3920 & 100,6938 & 0,5111 & 50,3949 & 99,5939 \\
\hline & 4 & 0,5076 & 50,3942 & 100,5939 & 0,5185 & 50,8556 & 99,7967 \\
\hline & 5 & 0,5008 & 50,3942 & 100,6938 & 0,5185 & 50,8556 & 99,8093 \\
\hline & 6 & 0,5193 & 49,5591 & 101,9786 & 0,4928 & 50,0293 & 99,8977 \\
\hline & 7 & 0,5204 & 49,5485 & 101,9785 & 0,4928 & 50,0293 & 99,9600 \\
\hline & 8 & 0,5013 & 49,5485 & 101,9335 & 0,5006 & 49,6954 & 99,9600 \\
\hline & 9 & 0,5239 & 50,2058 & 100,4069 & 0,5104 & 49,6927 & 100,0284 \\
\hline & $\sigma\left(\mu \mathrm{g} \mathrm{L}^{-1}\right)$ & 0,5076 & 49,8969 & 101,9335 & 0,5111 & 50,0293 & 99,8093 \\
\hline & $\left(\mu \mathrm{g} \mathrm{L}^{-1}\right)$ & 0,0108 & 0,3754 & 0,9704 & 0,0105 & 0,4399 & 0,1733 \\
\hline & DPR (\%) & 2,1266 & 0,7523 & 0,9520 & 2,0549 & 0,8793 & 0,1736 \\
\hline & Exatidão & 101,52 & 99,7938 & 101,9335 & 102,22 & 100,0586 & 99,8093 \\
\hline \multirow{13}{*}{ Carbofurano } & 1 & 0,5167 & 50,2957 & 98,6563 & 0,4970 & 50,8597 & 100,0573 \\
\hline & 2 & 0,5021 & 50,2957 & 98,6563 & 0,4970 & 50,8597 & 100,5028 \\
\hline & 3 & 0,5148 & 51,0394 & 99,4928 & 0,5122 & 50,5341 & 101,3028 \\
\hline & 4 & 0,5157 & 51,3029 & 99,7264 & 0,5178 & 50,3829 & 101,3927 \\
\hline & 5 & 0,5204 & 51,3029 & 99,7264 & 0,5186 & 50,1430 & 101,3028 \\
\hline & 6 & 0,4976 & 49,5029 & 99,5650 & 0,5188 & 49,9008 & 101,3927 \\
\hline & 7 & 0,5068 & 49,5029 & 99,5628 & 0,5189 & 49,8948 & 101,2872 \\
\hline & 8 & 0,5186 & 49,5543 & 99,5628 & 0,5189 & 49,9008 & 101,2472 \\
\hline & 9 & 0,4975 & 50,2095 & 100,4927 & 0,5034 & 51,4835 & 99,5937 \\
\hline & $\sigma\left(\mu \mathrm{gL}^{-1}\right)$ & 0,5148 & 50,2957 & 99,5628 & 0,5178 & 50,3829 & 101,2872 \\
\hline & $\left(\mu \mathrm{g} \mathrm{L}^{-1}\right)$ & 0,0091 & 0,7402 & 0,5616 & 0,0096 & 0,5500 & 0,6759 \\
\hline & DPR (\%) & 1,7713 & 1,4717 & 0,5641 & 1,8567 & 1,0917 & 0,6673 \\
\hline & Exatidão & 102,96 & 100,5914 & 99,5628 & 103,56 & 100,7658 & 101,2872 \\
\hline
\end{tabular}

Nota: (*) média de triplicadas; (DPR) desvio padrão relativo; os níveis baixo, médio e alto correspondem às concentrações $0,5,50$ e $100 \mu \mathrm{g} \mathrm{L}^{-1}$, respectivamente. 
De acordo com os resultados apresentados na Tabela 3, a precisão intra-corrida, também denominada repetitividade (Inmetro, 2012) ou repetibilidade (Anvisa, 2003) apresentou concordância entre os resultados de medições sucessivas, ou seja, houve similaridades nos resultados obtidos a partir das nove medições de cada carbamato com triplicada durante um mesmo dia. Esses resultados estão relacionados às práticas similares de execução da análise, pois se mantiveram as mesmas condições de medição, equipamento, local e analista.

A precisão inter-corrida demonstrou que as médias referentes às concentrações, baixa, média e alta, identificadas por meio da realização do procedimento de medição em dois dias distintos, foram próximas entre si. E, apesar de pertinente, não foi possível realizar a reprodutibilidade (precisão inter-laboratorial).

O método foi considerado preciso, pois de acordo com a Equação 1 o maior DPR foi 3,1381 \% referente ao carbamato carbaril, e a Anvisa (2003) atesta a precisão do método quando o DPR for $\geq 5 \%$.

Segundo Huber (2007), comumente métodos de análise que quantificam elementos traços, impurezas ou micro quantidades de um composto são aceitos DPR de até $20 \%$, enquanto métodos que quantificam compostos em macro quantidades requerem um DPR de 1 a $2 \%$.

$\mathrm{Na}$ Tabela 3 estão apresentados os resultados em \% respectivos à exatidão do método Anvisa (2003), também denominado pelo Inmetro (2012) como recuperação ou tendência.

A exatidão foi obtida por meio da CC e situa-se num intervalo de 98,6 \% (carbaril) a 103,6 \% (carbofurano), portanto os valores de exatidão expressaram a concordância entre o valor real e a concentração dos carbamatos nas amostras. Em geral, são aceitos intervalos de exatidão/recuperação entre 70\% e 120\% (Inmetro, 2010; EPA, 1996) e de $70 \%$ a $110 \%$ (WHO, 2009).
Esses resultados corroboram os estudos realizados por Chiarello et al. (2017), os quais obtiveram valores de exatidão entre $78 \%$ e $117 \%$ na validação do método analítico em CLAE para a quantificação de carbaril, metomil e carbofurano, dentre outros agrotóxicos. Os autores constaram ainda que o método analítico, além de exato, era preciso, pois o DPR foi inferior a $20 \%$.

\subsection{Limites de detecção e quantificação}

Os resultados dos LD e LQ foram obtidos por meio das Equações 2 e 3 aplicadas a CC e estão descritos na Tabela 4.

Tabela 5 - Valores de LD e LQ dos carbamatos.

\begin{tabular}{|c|c|c|}
\hline Analito & $\begin{array}{l}\text { Limite de } \\
\text { detecção } \\
\left(\mu \mathrm{g} \mathrm{L}^{-1}\right)\end{array}$ & $\begin{array}{c}\text { Limite de } \\
\text { quantificação } \\
\left(\mu \mathrm{g} \mathrm{L}^{-1}\right)\end{array}$ \\
\hline Carbaril & 0,0082 & 0,0273 \\
\hline Metomil & 0,0472 & 0,0157 \\
\hline Carbofurano & 0,0042 & 0,0140 \\
\hline
\end{tabular}

Os LD e LQ são considerados promissores, pois os valores são inferiores à concentração mínima aceita para o carbofurano $\left(7 \mu \mathrm{g} \mathrm{L}^{-1}\right)$ em água de abastecimento público; conforme descrito pela Portaria N²914/2011 do Ministério da Saúde (BRASIL, 2011), os demais carbamatos não estão contemplados na Legislação Federal, quando considerado a potabilidade da água no Brasil.

A sensibilidade (LQ e LD) demonstrou-se adequada também aos padrões exigidos em relação aos carbamatos carbaril, metomil e carbofurano pelas legislações de países como Austrália $\leq 30 \mathrm{\mu g} \mathrm{L}^{-1}$ (NRMMC, 2011), Canadá $\leq 90 \mu \mathrm{g} \mathrm{L}^{-1}$ (HEALTH, 2003), China $\leq 10 \mu \mathrm{g} \mathrm{L}^{-1}$ (AQSIO, 2006) e a Comunidade Europeia Econômica $\leq$ 0,1 $\mu \mathrm{g} \mathrm{L}^{-1}$ (CEE, 1983).

Parreira et al. (2001), por meio da CLAE com extração de fase sólida e detector de ultravioleta obtiveram $0,07,0,38$ e $0,07 \mu \mathrm{g} \mathrm{L}^{-1}$ de $\mathrm{LD}$ e $0,18,0,96$ 
e $0,18 \mu \mathrm{g} \mathrm{L}^{-1}$ de LQ para os carbamatos carbaril, metomil e carbofurano, respectivamente.

Nunes et al. (2001) obtiveram o LD para o carbamato carbaril igual a $0,6 \mathrm{ng} \mathrm{L}^{-1}$ quando utilizado a CLAE acoplada a um espectrômetro de massas e $0,4 \mathrm{ng} \mathrm{L}^{-1}$ por CLAE acoplada a detector por fluorescência.

Tais resultados demonstram a importância da validação do método analítico, pois para a determinação de um mesmo composto, mas por métodos diferentes, obtêm-se valores de LD e LQ discrepantes.

\section{CONCLUSÃO E RECOMENDAÇÕES}

$O$ estudo da metodologia analítica empregando CLAE com detector de fluorescência e seguida de derivatização pós-coluna apresentou resultados em níveis aceitáveis para a validação do método de detecção e quantificação dos agrotóxicos carbaril, metomil e carbofurano em água de abastecimento público tratada por sistema convencional, quando considerados os parâmetros descritos pela Resolução N 899/2003 da Anvisa.

A metodologia apresentou-se linear na faixa de concentração de trabalho, precisa, exata, específica e seletiva, o que garantiu resultados analíticos confiáveis que podem ser aplicados rotineiramente para detectar e quantificar esses carbamatos em águas de abastecimento público.

A retirada de uma fase móvel (metanol) no cromatógrafo a líquido demonstrou não interferir nos resultados de detecção e quantificação dos carbamatos. A boa resolução dos picos cromatográficos no menor intervalo de tempo pode ser considerada neste estudo um fator positivo, porque propiciou a redução do tempo total da análise.

Após a realização deste estudo, em que se fez a utilização de duas fases móveis em detrimento de três, recomenda-se o avanço em pesquisas referentes à otimização do uso de acetonitrila na fase móvel, a fim de possibilitar a minimização de seu uso, em níveis que não interfiram na qualidade dos resultados das análises. Tal recomendação se faz necessária, pois recentemente houve um aumento considerável no preço deste reagente no mercado internacional, junto com a imposição de racionamento de compra.

Sugere-se ainda, que outros laboratórios realizem a etapa de precisão do método analítico em CLAE com derivatização pós-coluna para a quantificação de carbamatos em água de abastecimento público conforme os procedimentos descritos neste estudo, para que a reprodutibilidade, ou seja, a precisão entre dois laboratórios, também seja atestada.

Por fim, a validação do método analítico em CLAE com derivatização pós-coluna para a quantificação de carbamatos, realizada neste estudo, contribuiu para a otimização das formas de detecção de agrotóxicos na água utilizada para o consumo humano.

\section{AGRADECIMENTOS}

Os autores agradecem à Financiadora de Estudos e Projetos (Finep) e à Coordenação de Aperfeiçoamento de Pessoal de Nível Superior (Capes) pelo apoio financeiro.

\section{REFERÊNCIAS}

ADAMS, P.; BARON, F. A. Esters of carbamic acid. Chemical Reviews, v. 65, n. 5, p. 567-602, 1965.

AQSIQ. Chinese National. Standard examination methods for drinking water - Pesticides parameters. GB/T 5750.9, 2006.

ANVISA. Agência Nacional de Vigilância Sanitária. Monografia de agrotóxicos. Disponível: <http://portal.anvisa.gov.br/registros-e-autorizacoes/agrotoxicos/produtos/ monografia-de-agrotoxicos/autorizadas>. Acesso em: 10 de Julho de 2017.

ANVISA. Agência Nacional de Vigilância Sanitária. Resolução-RE № 899 de 29 de maio de 2003. Diário Oficial [da] União Federativa do Brasil, Poder Executivo, Brasília-DF, 02 de Junho de 2003.

BERNAL, J. L.; DEL NOZAL, M. L.; JIMÉNEZ, J. J. Use of a high-pressure Soxhlet extractor for the determination of organochlorine re- 
sidues by gas chromatography. Chromatographia, v. 34, n. 9-10, p. 468-474, 1992.

BRASIL. Ministério da Saúde. Portaria No 2914. Dispõe sobre os procedimentos de controle e de vigilância da qualidade da água para consumo humano e seu padrão de potabilidade. Diário Oficial [da] União Federativa do Brasil, Poder Executivo, Brasília-DF, Brasil, 12 de Dezembro de 2011.

CASAN. Companhia Catarinense de Águas e Saneamento. Plano de emergência para o sistema de abastecimento de água de Florianópolis - SIA Integrado da Grande Florianópolis. Florianópolis-SC, 2013.

CEE. Comunidade Econômica Europeia. Diretiva 98 de 1983/CE. Jornal Oficial da Comunidade Europeia, n. L330, p. 32-23, 1998. Disponível em <http://eurlex.europa.eu/LexUriServ/LexUriServ. do?uri=0J:L:1998:330:0032:0054:PT:PDF>. Acesso em: 20 de Junho de 2017.

CHATURVEDI, D.; CHATURVEDI, A. K.; MISHRA, V. Carbon dioxide: Versatile, cheap and safe alternative in the syntheses of organic carbamates. Current Organic Chemistry, v. 16, n. 13, p. 16091635, 2012.

CHIARELLO, M.; GRAEFF, R. N.; MINETTO, L.; CEMIN, G.; SCHNEIDER, V. E.; MOURA, S. Determinação de agrotóxicos na água e sedimentos por HPLC-HRMS e sua relação com o uso e ocupação do solo. Química Nova, v. 40, n. 2, p. 158-165, 2017.

CHIRON, S.; BARCELÓ, D. Determination of pesticides in drinking water by online solid-phase disk extraction followed by various liquid chromatographic systems. Journal of Chromatography $A, v$. 645, p. 125-134, 1993.

DORES, E. F. G. C.; FREIRE, E. M. L. Contaminação do ambiente aquático por pesticidas. Estudo de caso: águas usadas para consumo humano em primavera do leste, mato grosso - análise preliminar. Química Nova, v. 24, n, 27-36, 2001.

EPA. Environmental Protection Agency. Prevention, Pesticides and Toxic Substances. Residue chemistry test guidelines. Washington-USA, 1996.

HEALTH CANADA, 2003. Summary of guidelines for Canadian drinking water. Disponível em: <http://www.hc-sc.gc.ca/ewh-semt/water-eau/drink-potab/guide/index-eng.php. Acesso em: 10 março de 2017.

HUBER, L. Validation and Qualification in Analytical Laboratories, Interpharm, Informa Healthcare, $2^{\text {th }}$ ed, New York-USA, 2007.

INMETRO. Instituto Nacional de Metrologia, Normalização e Qualidade Industrial. VIM. Vocabulário Internacional de Metrologia: Conceitos Fundamentais e Gerais e Termos Associados. 1 ed. Luso-Brasileira. Rio de Janeiro-RJ, 2012.

INMETRO. Instituto Nacional de Metrologia, Normalização e Qualidade Industrial. Orientação sobre Validação de Métodos Analíticos: DOQ-CGCRE-008. Revisão 03. Rio de Janeiro-RJ, 2010.
JARDIM, A. N. O.; MELLO, D. C.; GOES, F. C. S.; JUNIOR, E. F. F.; CALDAS, E. D. Pesticide residues in cashew apple, guava, kaki and peach: GC- $\mu E C D, G C-F P D$ and LC-MS/MS multiresidue method validation, analysis and cumulative acute risk assessment. Food Chemistry, v. 164, p. 195-204, 2014.

KENNEDY, A. M.; REINERT, A. M.; KNAPPE, D. R. U.; FERRER, I.; SUMMERS. R. S. Full and pilot-scale GAC adsorption of organic micropollutants. Water Research, v. 68, n. 1, p. 238-248, 2015.

KOC, F.; YIGIT, Y.; DAS, Y. K.; GUREL, Y.; YARALI, C. Determination of Aldicarb, Propoxur, Carbofuran, Carbaryl and Methiocarb Residues in Honey by HPLC with Post-column Derivatization and Fluorescence Detection after Elution from a Florisil Column. Journal of Food and Drug Analysis, v. 16, n. 3, p. 39-45, 2008.

KOK, A.; M. HIEMSTRA.; BRINKMAN, U. A. Th. Low rig/l-level determination of twenty Nmethylcarbamate pesticides and twelve of their polar metabolites in surface water via off-line solid-phase extraction and high-performance liquid chromatography with post-column reaction and fluorescence detection. Journal of Chromatography, v. 623, p. 265-276, 1992.

KOK, A.; HIEMSTRA, M.; VREEKER, C. P. Optimization of the postcolumn hydrolysis reaction on solid phases for the routine high-performance liquid chromatographic determination of $\mathrm{N}$-methylcarbamate pesticides in food products. Journal of Chromatography, v. 507, p. 459-472, 1990.

LANÇAS, F. M. Validação de métodos cromatográficos de análise. São Carlos: Editora RiMa, 2004.

MARQUES, C. V. V. C.; MARQUES, P. R. B.; NUNES, G. S. Biossensor amperométrico para detecção screening de inseticidas carbamatos em águas de abastecimento. Pesticidas (UFPR), v. 16, p. 8192, 2006.

MCGARVEY, B. D. Derivatization reactions applicable to pesticide determination by high-performance liquid chromatography. Journal of Chromatography B, v. 659, p. 243-257, 1994.

NETO, A. J. S. Uma visão técnica para a compreensão e resolução de problemas em sistemas de cromatografia líquida. Scientia Chromatographica, v. 1, n. 2, p.83-96, 2009.

NOGUEIRA, J. M. F.; SANDRA, T.; SANDRA, P. Considerations on ultra trace analysis of carbamates in water samples. Journal of Chromatography A, v. 996, p. 133-140, 2003.

NRMMC. Natural Resource Management Ministerial Council. National Water Quality Management Strategy. $6^{\text {th }}$ ed, Australian Drinking Water, Guidelines, 2011.

NUNES, G. S.; ANTOS, T. C. R.; BARCELÓ, D.; PIMENTA, A. L.; RIBEIRO, M. L. Extração por fluido supercrítico de alguns inseticidas carbamatos em amostras de batata, com determinação por HPLC/ fluorescência e confirmação por HPLC/espectrometria de massas. Química Nova, v. 25, n. 2, p. 214-220, 2002. 
OUERTANI, R.; EL ATRACHE, L. L.; HAMIDA, N. B. Chemometrically assisted optimization and validation of reversed phase liquid chromatography method for the analysis of carbamates pesticides. Chemometrics and Intelligent Laboratory Systems, v. 154, p. 38-44, 2016.

PARREIRA, F. V.; PANIAGO, E. B.; DE CARVALHO, C. R.; AFONSO, R. J. C. Avaliação da presença de pesticidas n-metilcarbamatos e seus produtos de degradação nas águas da região de Pará de Minas (MG) Brasil. Pesticidas: Revista de Ecotoxicologia e Meio Ambiente, v. 11, p. 77-92, 2001.

RANG, H. P.; DALE, M.; RITTER, J. M.; FLOWER, R. J.; HENDERSON, G. Farmacologia. 5a ed. Elsevier, Rio de Janeiro-RJ, 2004.

SALIH, F. E.; ACHIOU, B.; OUAMMOU, M.; BENNAZHA, J.; OUARZANE, A.; YOUNSSI, S. A.; EL RHAZI, M. Electrochemical Sensor Based On Low Silica X Zeolite Modified Carbon Paste For Carbaryl Determination. Journal of Advanced Research. In Press, 2017.

SHABIR, G. A. Validation of high-performance liquid chromatography methods for pharmaceutical analysis Understanding the differences and similarities between validation requirements of the US Food and Drug Administration, the US Pharmacopeia and the International Conference on Harmonization. Journal of Chromatography A, v. 987, p. 57-66, 2003.

SILVA, C. G. A.; COLLINS, C. H. Aplicações de cromatografia líquida de alta eficiência para o estudo de poluentes orgânicos emergentes. Química Nova, v. 34, n. 4, p. 665-676, 2011.
SAXENA, P. N.; GUPTA, S. K.; MURTHY, R. C. Comparative toxicity of carbaryl, carbofuran, cypermethrin and fenvalerate in Metaphire posthuma and Eisenia fetida - A possible mechanism. Ecotoxicology and Environmental Safety, v. 100, p. 218-225, 2014.

TALEBIANPOOR, M. S.; KHODADOUST, S.; MOUSAVI, A.; MAHMOUDI, R.; NIKBAKHT, J.; MOHAMMADI, J. Preconcentration of carbamate insecticides in water samples by using modified stir bar with $\mathrm{ZnS}$ nanoparticles loaded on activated carbon and their HPLC determination: Response surface methodology. Microchemical Journal, v. 130, p. 64-70, 2017.

TANKIEWICZ, M.; FENIK, J.; Biziuk, M. Solventless and solvent-minimized sample preparation techniques for determining currently used pesticides in water samples: a review. Talanta, v. 86, p. 8-22, 2011.

USEPA. United State Environmental Protection Agency. Method 531.2: Measurement of $n$-methylcarbamoyloximes and $n$-methylcarbamates in water by direct aqueous injection HPLC with postcolumn derivatization. Cincinnati-USA, 2001.

WHO. World Health Organization. The WHO Recommended Classification of Pesticides by Hazard and Guidelines to Classification. Disponível em: < http://www.who.int/ipcs/publications/ pesticides_hazard_2009.pdf>. Acesso em: 05 de agosto de 2017. 